\title{
The transition between Neumann and Dirichlet boundary conditions in isotropic elastic plates
}

\author{
R. R. Moukhomodiarov* \\ Department of Mathematical Theory of Elasticity and Biomechanics, \\ Saratov State University, 83 Astrakhanskaya St., Saratov 410026, Russia. \\ A. V. Pichugin \\ Department of Mathematical Sciences, SISCM, \\ Brunel University, Uxbridge, Middlesex, UB8 3PH, UK. \\ and G. A. Rogerson ${ }^{\dagger}$ \\ School of Computing and Mathematics, Keele University, \\ Keele, Newcastle, Staffordshire, ST5 5BG, UK.
}

\begin{abstract}
The transition from Neumann (traction-free) to Dirichlet (fixed-face) boundary conditions is investigated in respect of wave propagation in a linear isotropic elastic layer. Attention is focused on the implications of such a transition on the dispersion curve branches within the long wave region. The formation of low frequency band gap that is expected to exist in layers with Dirichlet boundary condition is shown to be caused by different mechanisms in anti-symmetric and symmetric cases. Certain implications to short-wave propagation in the layer are also investigated. The study includes both a numerical investigation and a multi-parameter asymptotic analysis.
\end{abstract}

\section{Introduction}

Most structures are utilized within scenarios in which they are surrounded by a medium that interacts with them. When the contrast in acoustic properties between the medium and structure is large, it is often possible to disregard the influence of the surrounding medium and significantly simplify the model. In particular, stress-free (Neumann) boundary

\footnotetext{
*Emails: rinatm@rambler.ru, aleksey.pichugin@brunel.ac.uk, g.a.rogerson@maths.keele.ac.uk

${ }^{\dagger}$ Author for correspondence.
} 
conditions are not only valid for elastic bodies in vacuum, but also serve as a very good approximation to the behaviour of many solids in air. Similarly, a relatively soft body in a stiff enclosure may be well approximated by a fixed (Dirichlet) boundary conditions. Clearly, there is a wide range of situations laying in between these two extremes and it is often unfeasible to model the environment of a structure in minute detail. For example, the boundary condition appropriate for the inner or outer wall of a human blood vessel cannot truly be described as either "free" or "fixed"; correct modelling of surrounding tissue is still a daunting prospect.

In acoustics this situation is often resolved by considering so-called impedance boundary conditions that, as impedance grows, provide a natural transition from Neumann to Dirichlet boundary conditions. Intuitively, we can expect that in the elastic case a good first approximation can be obtained by assuming that the field at its boundaries satisfies a Hooke-type law, i.e. that the boundaries are elastically restrained. While the idea appears natural, surprisingly little research has been devoted to wave propagation in bodies with such boundaries. As an exception, Mindlin [8] looked into wave propagation in an isotropic elastic layer with boundaries elastically restrained in the normal direction; however, he was only interested in analysing a grid of bounds for dispersion curves. It is our aim to extend Mindlin's study and investigate more general aspects of wave propagation in layers with elastically restrained boundaries, with particular emphasis on long-wave propagation.

Section 2 is devoted to the physical motivation behind the elastically restrained boundary conditions (ERBC). The general formulation of ERBC in the two-dimensional case implies introduction of four additional parameters into the problem. We limit our analysis to situations for which the boundary conditions are the same on both the top and bottom faces; such symmetric geometry reduces the number of additional parameters to two and enables splitting of the problem into two simpler cases, so-called anti-symmetric and symmetric motion.

In Section 3 we derive the dispersion relations that generalise classical Rayleigh [12] and Lamb [7] result to the isotropic elastic layer with ERBC. In order to further reduce the considered parameter space, much of the subsequent analysis is concerned with particular types of ERBC, depending on a single parameter. The case that was previously considered by Mindlin [8] is given a particularly detailed treatment.

Waveguides with Dirichlet boundary conditions are known to act as high-pass filters, i.e. they cannot propagate waves below a certain cut-off frequency. The same is true for elastic layers with fixed faces. On the other hand, the layers with free faces possess at least one propagating mode for all frequencies. In Section 4 we demonstrate in some detail how the introduction of elastic support leads to the emergence of a low-frequency band gap 
in both the symmetric and anti-symmetric dispersion relations. The associated long-wave asymptotic regimes are identified and analysed in Section 5 for a particular kind of ERBC.

Generally, it is possible to identify two types of long-wave motions in elastic layers: lowfrequency and high-frequency, see [3]. The long-wave low-frequency asymptotics describe so-called fundamental modes, relating to the classical approximate theories of rods, plates and shells, as well as their modern generalisations, see e.g. $[5,13,11]$. The long-wave high-frequency asymptotics correspond to "thickness vibrations", long-wave limits of higher solution modes. This type of motion is particularly important for layers with fixed faces, where the classical long-wave low-frequency mode does not exist, see [2].

Long-wave high-frequency motion of elastic layers has previously been analysed for the variety of anisotropic and pre-stressed media, see for example $[4,10,6]$. A particularly interesting aspect of such motions concerns the change in asymptotic structure of the expansions that happens when the long-wave limits of two different modes coincide, see [15]. In this case it is necessary to develop a separate asymptotic process, described in $[9,14]$. The resulting asymptotics are not uniform with respect to the parameters affecting the long-wave high frequency limits. In this paper we present a procedure capable of deriving long-wave high-frequency expansions that are uniform in this respect.

The paper is concluded in Section 6 by some remarks on the short-wave limiting behaviour of the derived dispersion relations. We observe that the two coupled Rayleigh wave travelling along the opposite faces, the expected short-wave limit in isotropic elastic layers, are not immediately destroyed by the presence of elastic support at the boundary and, in fact, exist for any finite value of the boundary elastic modulus. However, they become dispersive and, as the boundary gets stiffer, degenerate into a surface-skimming shear wave at a particular wave number.

\section{Modelling elastically restrained boundary}

Consider an infinite isotropic elastic layer of thickness $2 h$ in a state of plane strain that is free from external forces. Classical boundary conditions on the faces of the layer typically belong to two types: the free face boundary condition, obtained by setting surface tractions to zero, and so-called fixed face boundary condition implying the vanishing of the displacements. A combination of these two conditions is sometimes used, in which one of the tractions is required to vanish along with one of the displacements. This type of boundary condition is usually termed mixed, and its importance is related to the fact that it enables splitting the vector elasticity problem into two scalar problems, see [8] or [1].

All of these boundary conditions are limiting cases that are not usually met in reality, 


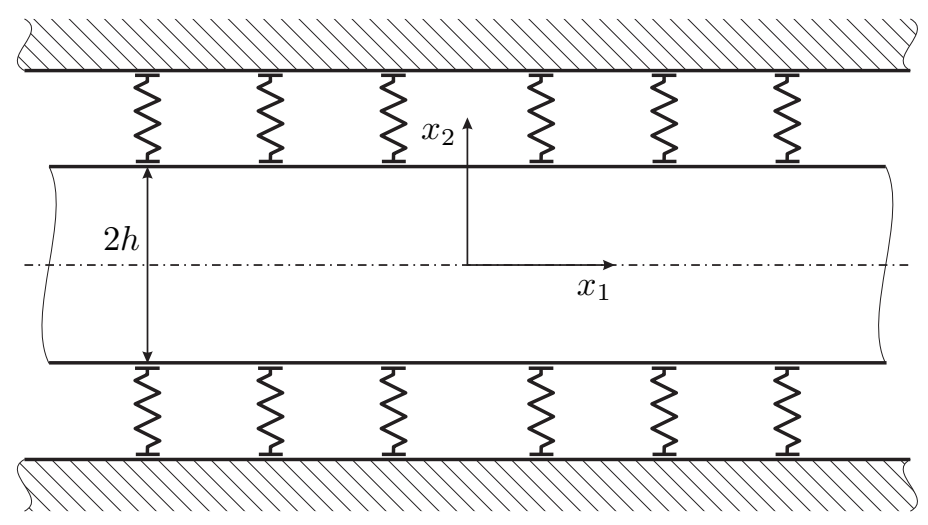

Figure 1: Elastic restraint in the normal direction.

because the free face conditions mean that the layer is in vacuum, whereas the fixed face conditions describe the layer embedded in an infinitely stiff enclosure. Classical boundary conditions are often very good approximations, but sometimes it is necessary to take into account more complex interactions at the boundary. Generally, we may introduce ERBC as

$$
\tau_{21}+d_{1}^{ \pm} u_{1}=0, \quad \tau_{22} \pm d_{2}^{ \pm} u_{2}=0, \quad x= \pm h
$$

so that when $d_{n}^{ \pm} \rightarrow 0, n=1,2$, we arrive at the free faces boundary conditions and when $d_{n}^{ \pm} \rightarrow \infty, n=1,2$, the fixed faces case is obtained. However, it is difficult to analyse solutions that may depend on four additional elastic constants $d_{n}^{ \pm}, n=1,2$. The analysis may be simplified by assuming that the elastic modulae at the top face are the same as the ones at the bottom, i.e. $d_{n}^{+}=d_{n}^{-}=d_{n}, n=1,2$. Such a layer possesses the reflectional transverse symmetry, which enables reducing the general problem of wave propagation to two simpler problems dealing with propagation of purely anti-symmetric or symmetric waves.

More insightful analysis is possible if we restrict our attention to certain types of ERBC that are characterised by a single parameter. The first type is associated with an infinite layer that is held between perfectly lubricated elastic surfaces, see Figure 1. This can be modelled by considering uniformly distributed linearly elastic springs that resist normal displacements at the layer faces, but produce no tangential restraint, for which we assumed boundary condition in the form

$$
\tau_{21}=0, \quad \tau_{22} \pm d u_{2}=0, \quad \text { where } \quad x_{2}= \pm h .
$$

These conditions were previously considered by Mindlin [8]. As boundary, as we allow the modulus $d$ to increase from 0 to $\infty$, the conditions at the layer faces undergo transition from free to mixed. A subsequent transition to fixed boundary conditions may be modelled by 


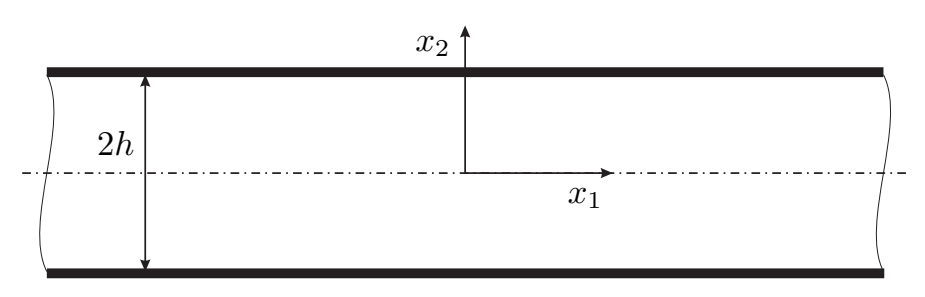

Figure 2: Elastic restraint in the tangential direction.

considering

$$
\tau_{21}+d u_{1}=0, \quad u_{2}=0, \text { where } x_{2}= \pm h
$$

Alternatively, we may start by assuming that our layer has a very thin flexible membrane attached to its faces, which produces no restraint in the normal direction, see Figure 2. This can be described by the boundary conditions of the form

$$
\tau_{21}+d u_{1}=0, \quad \tau_{22}=0, \quad \text { where } \quad x_{2}= \pm h .
$$

A very soft membrane results in the free-faces case, while an inextensible membrane precludes any tangential displacements on the faces. A subsequent transition to fixed boundary conditions may be modelled by considering

$$
u_{1}=0, \quad \tau_{22} \pm d u_{2}=0, \quad \text { where } \quad x_{2}= \pm h
$$

The principal advantage of using such a single-parameter boundary conditions $(2.2)-(2.5)$ is that it enables the transition from free to fixed faces through two well-understood cases of mixed boundary conditions. This will enable us to perform very detailed analysis of mode transition from free to fixed faces, as well as formulate a natural physical interpretation of this process.

Each of the boundary conditions (2.2)-(2.5) may be obtained by appropriate specialisation of the elastic constants in (2.1). Thus, we intend to derive dispersion relations for the most general case and then analyse specific single-parameter cases by appropriately specialising the boundary modulae. This process may in fact be performed more conveniently if the boundary conditions at $x_{2}= \pm h$ are given by

$$
\begin{aligned}
& \frac{1}{\mu}\left(\alpha_{1} \tau_{21}+\delta_{1} \frac{\mu}{h} u_{1}\right)=\alpha_{1}\left\{\frac{\partial u_{1}}{\partial x_{2}}+\frac{\partial u_{2}}{\partial x_{1}}\right\}+\frac{\delta_{1}}{h} u_{1}=0 \\
& \frac{1}{\rho}\left(\alpha_{2} \tau_{22} \pm \delta_{2} \frac{\mu}{h} u_{2}\right)=\alpha_{2}\left\{\left(c_{1}^{2}-2 c_{2}^{2}\right) \frac{\partial u_{1}}{\partial x_{1}}+c_{1}^{2} \frac{\partial u_{2}}{\partial x_{2}}\right\} \pm c_{2}^{2} \frac{\delta_{2}}{h} u_{2}=0
\end{aligned}
$$

where $\alpha_{n}, \delta_{n}, n=1,2$, are non-dimensional scalars, $c_{1}=\sqrt{(\lambda+2 \mu) / \rho}$ the dilatational wave speed, $c_{2}=\sqrt{\mu / \rho}$ the shear wave speed and $\lambda$ and $\mu$ the Lamé parameters. The free faces boundary conditions are obtained by selecting $\alpha_{1}=\alpha_{2}=1$ and $\delta_{1}=\delta_{2}=0$. The fixed faces are obtained when $\alpha_{1}=\alpha_{2}=0$ and $\delta_{1}=\delta_{2}=1$. 


\section{Dispersion relations}

Consider a layer composed of linear isotropic elastic material subjected to the boundary conditions (2.6)-(2.7). The equations of motion may be presented in displacement form as follows

$$
\begin{aligned}
& c_{1}^{2} \frac{\partial^{2} u_{1}}{\partial x_{1}^{2}}+\left(c_{1}^{2}-c_{2}^{2}\right) \frac{\partial^{2} u_{2}}{\partial x_{1} \partial x_{2}}+c_{2}^{2} \frac{\partial^{2} u_{1}}{\partial x_{2}^{2}}=\frac{\partial^{2} u_{1}}{\partial t^{2}}, \\
& c_{2}^{2} \frac{\partial^{2} u_{2}}{\partial x_{1}^{2}}+\left(c_{1}^{2}-c_{2}^{2}\right) \frac{\partial^{2} u_{1}}{\partial x_{1} \partial x_{2}}+c_{1}^{2} \frac{\partial^{2} u_{2}}{\partial x_{2}^{2}}=\frac{\partial^{2} u_{2}}{\partial t^{2}} .
\end{aligned}
$$

We seek solutions of (3.1)-(3.2) in the form of travelling harmonic waves

$$
u_{n}\left(x_{1}, x_{2}, t\right)=U_{n} e^{k q x_{2}} e^{\mathrm{i} k\left(x_{1}-v t\right)}, \quad n=1,2,
$$

where $k$ is the longitudinal wave number and $v$ the phase speed, $\mathrm{i} k q$ is the transverse wave number that is chosen to ensure that the equations of motion (3.1), (3.2) have non-trivial solutions. Substituting (3.3) into (3.1), (3.2) and requiring the resulting linear homogeneous system to have non-trivial solution, delivers a quadratic equation in $q^{2}$, with four roots given by

$$
q_{1}^{2}=1-V^{2}, \quad q_{2}^{2}=1-\frac{V^{2}}{\kappa^{2}},
$$

in which $V=v / c_{2}$ is the non-dimensional phase velocity, $\kappa^{2}=c_{1}^{2} / c_{2}^{2}=2(1-\nu) /(1-2 \nu)$ the ratio of longitudinal and shear wave speeds and $\nu$ Poisson's ratio. Any solution for $u_{1}$ and $u_{2}$ can be represented as a linear combination of the four integrals associated with roots (3.4). By referring back to the equations of motion these solutions can be given by

$$
\begin{gathered}
u_{1}\left(x_{1}, x_{2}, t\right)=\left\{A_{1} \cosh \left(k q_{1} x_{2}\right)+A_{2} \sinh \left(k q_{1} x_{2}\right)\right. \\
\left.+A_{3} \cosh \left(k q_{2} x_{2}\right)+A_{4} \sinh \left(k q_{2} x_{2}\right)\right\} e^{\mathrm{i} k\left(x_{1}-v t\right)}, \\
\begin{aligned}
u_{2}\left(x_{1}, x_{2}, t\right)=-\frac{\mathrm{i}}{q_{1}}\{ & A_{1} \sinh \left(k q_{1} x_{2}\right)+A_{2} \cosh \left(k q_{1} x_{2}\right) \\
- & \left.q_{1} q_{2}\left(A_{3} \sinh \left(k q_{2} x_{2}\right)+A_{4} \cosh \left(k q_{2} x_{2}\right)\right)\right\} e^{\mathrm{i} k\left(x_{1}-v t\right)} .
\end{aligned}
\end{gathered}
$$

Expressions (3.5)-(3.6) contain terms that are both symmetric and anti-symmetric with respect to $x_{2}$. Due to the symmetry of the boundary conditions it is possible to impose a symmetry requirement on (3.5)-(3.6) and then obtain the dispersion relation by satisfying boundary conditions on one face.

For anti-symmetric modes we require $A_{1}=A_{3}=0$ in (3.5), (3.6), with the associated dispersion relation taking the form

$$
\begin{aligned}
\left(4 q_{1} q_{2}-\frac{\delta_{1} \delta_{2}}{\alpha_{1} \alpha_{2}} \frac{q_{1} q_{2}}{\eta^{2}}+\frac{\delta_{1}}{\alpha_{1}} \frac{q_{1} V^{2} \tanh \left(\eta q_{2}\right)}{\eta}\right) \tanh \left(\eta q_{1}\right) \\
\quad+\left(\frac{\delta_{1} \delta_{2}}{\alpha_{1} \alpha_{2}} \frac{1}{\eta^{2}}-\left(2+V^{2}\right)^{2}\right) \tanh \left(\eta q_{2}\right)+\frac{\delta_{2}}{\alpha_{2}} \frac{q_{2} V^{2}}{\eta}=0,
\end{aligned}
$$



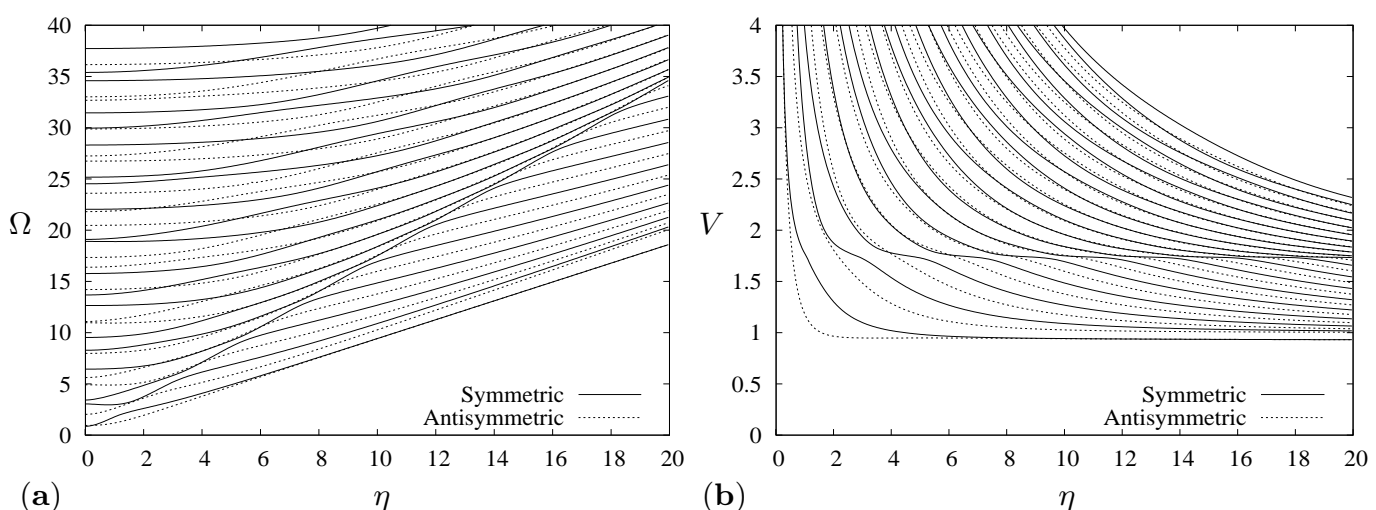

(b)

Figure 3: Scaled frequency (a) and phase speed (b) against wave number for the layer subjected to ERBC (2.6), (2.7) with $\alpha_{1}=\alpha_{2}=\delta_{1}=\delta_{2}=1$ and $\kappa=\sqrt{3}$.

where the non-dimensional wave number $\eta \equiv k h$. The dispersion relation for symmetric modes is obtained by putting $A_{2}=A_{4}=0$, with the result

$$
\begin{aligned}
&\left(4 q_{1} q_{2}-\frac{\delta_{1} \delta_{2}}{\alpha_{1} \alpha_{2}} \frac{q_{1} q_{2}}{\eta^{2}}+\frac{\delta_{1}}{\alpha_{1}} \frac{q_{1} V^{2} \operatorname{coth}\left(\eta q_{2}\right)}{\eta}\right) \operatorname{coth}\left(\eta q_{1}\right) \\
&+\left(\frac{\delta_{1} \delta_{2}}{\alpha_{1} \alpha_{2}} \frac{1}{\eta^{2}}-\left(2+V^{2}\right)^{2}\right) \operatorname{coth}\left(\eta q_{2}\right)+\frac{\delta_{2}}{\alpha_{2}} \frac{q_{2} V^{2}}{\eta}=0 .
\end{aligned}
$$

which is, essentially, (3.7) with all tanh replaced by coth. It can be easily verified that by setting $\alpha_{1}=\alpha_{2}=1$ and $\delta_{1}=\delta_{2}=0$ we arrive at the classical Rayleigh-Lamb frequency equations. When $\alpha_{1}=\alpha_{2}=0$ and $\delta_{1}=\delta_{2}=1$ the previously known dispersion relations for layer with fixed faces are recovered.

As an illustration, we have generated two plots with both symmetric and anti-symmetric dispersion curves for a typical set of material parameters with $\kappa=\sqrt{3}$, i.e. $\nu=1 / 4$. Figure 3(a) shows non-dimensional frequency against wave number, while Figure 3(b) shows non-dimensional phase velocity against wave number. Since all of the parameters $\alpha_{1}=$ $\alpha_{2}=\delta_{1}=\delta_{2}=1$, Figure 3 demonstrates the response of the layer subjected to boundary conditions that are intermediate between free faces and fixed faces. Evidently, there are no long-wave low-frequency modes present in this case.

If we were to fix the non-dimensional frequency $\Omega=V \eta$ and compute the long-wave limit of the anti-symmetric dispersion relation (3.7), the result simplifies into

$$
\left(\frac{\delta_{1}}{\alpha_{1}} \sin \Omega+\Omega \cos \Omega\right)\left(\kappa \Omega \sin \frac{\Omega}{\kappa}-\frac{\delta_{2}}{\alpha_{2}} \cos \frac{\Omega}{\kappa}\right)=O\left(\eta^{2}\right) .
$$

The same procedure applied to the symmetric dispersion relation (3.8) results in

$$
\left(\Omega \sin \Omega-\frac{\delta_{1}}{\alpha_{1}} \cos \Omega\right)\left(\frac{\delta_{2}}{\alpha_{2}} \sin \frac{\Omega}{\kappa}+\kappa \Omega \cos \frac{\Omega}{\kappa}\right)=O\left(\eta^{2}\right) .
$$



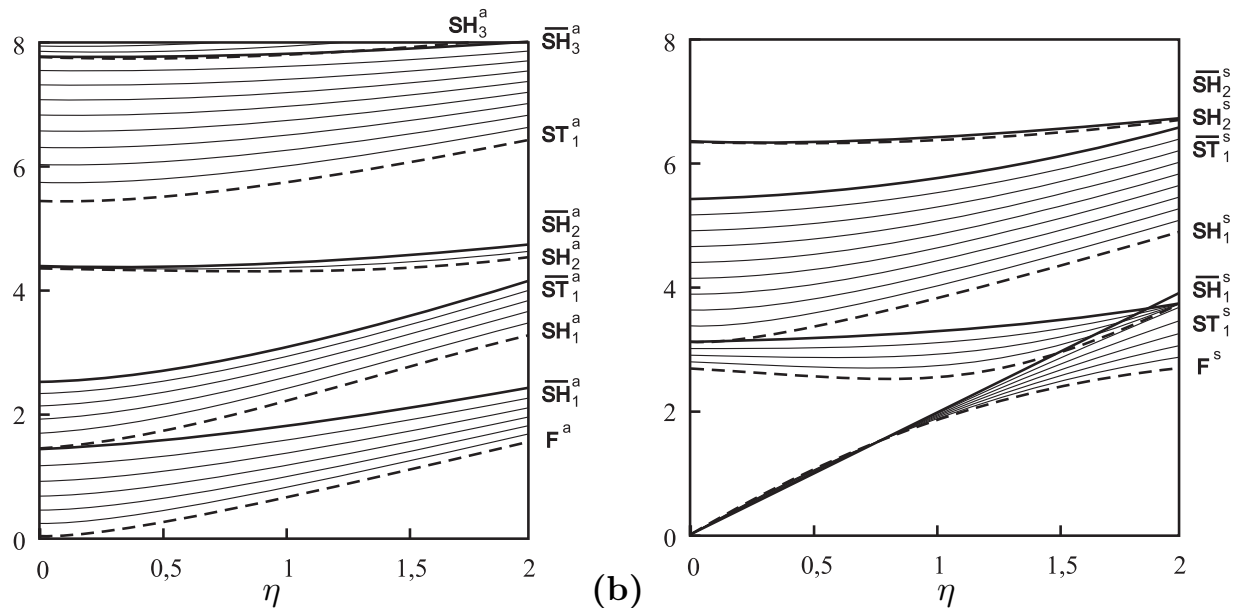

Figure 4: (a) anti-symmetric and (b) symmetric mode transformations in an elastic isotropic layer with boundary conditions (2.2), where $\kappa=\sqrt{3}$.

Evidently, all long-wave solutions can be classified into two families, corresponding to the individual factors within the leading order dispersion relations (3.9) and (3.10). The forthcoming analysis will demonstrate that these factors do in fact represent the thickness shear and thickness stretch resonance frequencies of our problem. The important point to note here is that each of these factors is only affected by a single pair of $\delta_{n}$ and $\alpha_{n}$. Thus, we can study the processes of long-wave mode transformation in full detail by individually analysing simple single-parameter types of ERBC.

\section{Numerical analysis of mode transformation}

It will now be helpful to investigate the transformation of both anti-symmetric and symmetric modes arising through the variation of boundary condition modulae. We begin by considering the configuration shown in Figure 1, with the corresponding boundary conditions given by (2.2). The appropriate dispersion relations for anti-symmetric and symmetric waves are obtained directly from (3.7) and (3.8) by inserting $\alpha_{1}=\alpha_{2}=1, \delta_{1}=0, \delta_{2}=\delta$.

The transformation of the first four anti-symmetric modes are presented in Figure 4(a). The dashed curves correspond to the free-faces case (i.e. $\delta=0$ ), with the thick solid lines corresponding to the mixed boundary conditions on the layer faces $(\delta \rightarrow \infty)$ and other solid lines transitional modes. Other designations used in this Section's diagrams are as follows: SH and ST denote higher harmonics originating from the thickness shear and thickness stretch long-wave limits, see [8]. The subscripts denote mode numbers, whereas superscripts 'a' and 's' indicate anti-symmetric and symmetric modes; the overbar is used to designate the modes in the case of mixed boundary conditions. For example, $\overline{S T}_{2}^{a}$ denotes the anti- 

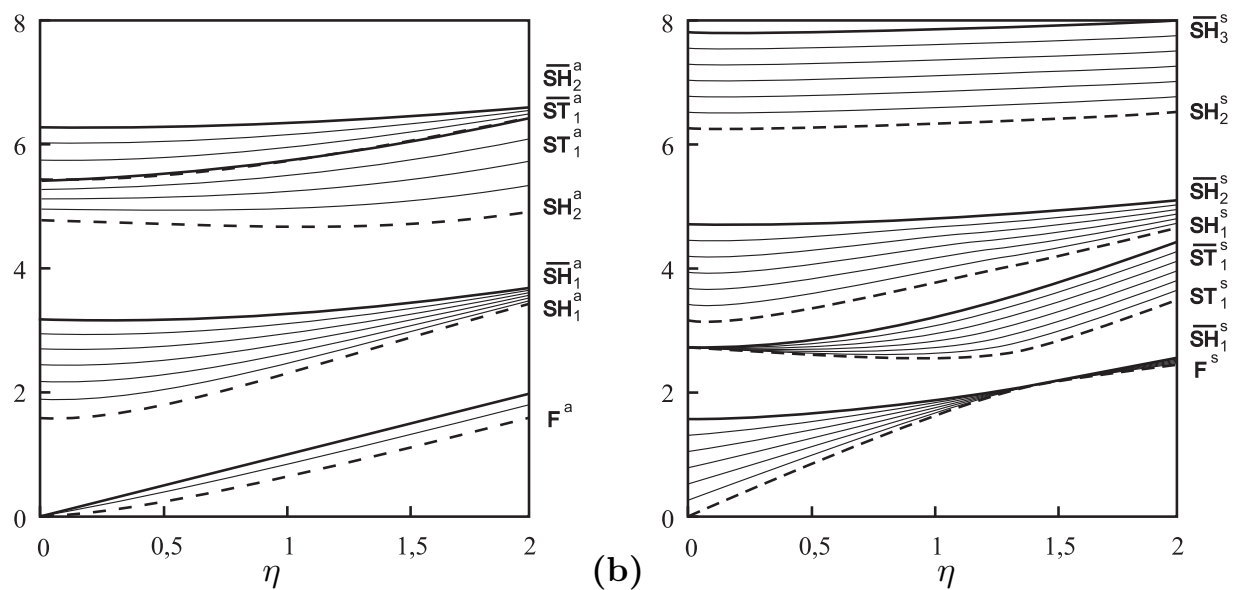

Figure 5: (a) anti-symmetric and (b) symmetric mode transformations in an elastic isotropic layer with boundary conditions $(2.4)$, where $\kappa=\sqrt{3}$.

symmetric mode that originates from the second thickness stretch resonance frequency for a layer with the mixed boundary conditions.

Let us now consider anti-symmetric mode transformations in a greater detail. As $\delta$ increases from 0 to $\infty$, the anti-symmetric fundamental mode $F^{a}$ moves smoothly to became harmonic $\overline{S T}_{1}^{a}$ originating from the first thickness stretch resonance frequency for the layer with mixed boundary conditions. This means that any boundary restraint in the normal direction leads to the formation of low-frequency band gap for anti-symmetric motions. All stretch resonance modes $S T_{n}^{a}$, with associated cut-off frequencies $\kappa n \pi$, transform into $S T_{n+1}^{a}$, with corresponding long-wave limits $\kappa(n+1 / 2) \pi$. As can be seen within figure $4(\mathrm{a})$, the cut-off frequencies of $S H$ modes are not affected. The general long-wave behaviour of $S H$ modes is not significantly influenced by $\delta$, see e.g. transition $S H_{2}^{a} \rightarrow \overline{S H}_{2}^{a}$. However, those $S H$ modes whose cut-off frequency $(n-1 / 2) \pi$ falls within the transition interval $[\kappa m \pi, \kappa(m+1 / 2) \pi]$ of the cut-off frequency of $m$ th $S T$ mode are affected more significantly, see $S H_{1}^{a} \rightarrow \overline{S H}_{1}^{a}$.

The transformations of the first four branches of the corresponding symmetric dispersion relation reveal a slightly different structure of the mode transition, see Figure 4(b). The fundamental mode of the layer with free faces $F^{s}$ becomes non-dispersive branch $V=1$ in the limit $\delta \rightarrow \infty$, as is expected from the layer with mixed boundary conditions, see [1]. The behaviour of symmetric harmonics is broadly similar to that seen in respect of the anti-symmetric case. Modes related to thickness shear originate from $\pi n$ and are only weakly affected by changes in $\delta$. The cut-off frequencies of modes associated with thickness stretch resonances vary between $\kappa(n-1 / 2) \pi$ and $\kappa n \pi, n=1,2, \ldots$

Mode transformations produced for boundary conditions (2.4), i.e. by selecting $\alpha_{1}=$ 
$\alpha_{2}=1, \delta_{1}=\delta, \delta_{2}=0$ are presented in Figure 5 . In this case, the anti-symmetric fundamental mode degenerates into the non-dispersive mode $V=1$ as $\delta \rightarrow \infty$. The cut-off frequency of the symmetric fundamental mode $F^{s}$ moves away from 0 , resulting in a low-frequency band gap and becoming the first harmonic $\overline{S H}_{1}^{s}$. More generally, the cut-off frequencies and mode transformations for boundary conditions (2.4) are similar to those for boundary conditions (2.2), switching the anti-symmetric and symmetric cases around. Accordingly, the cut-off frequencies of the $S T$-modes remain constant and the cut-off frequencies of $S H$ modes move smoothly to became the thickness shear resonance frequencies for a layer with the mixed boundary conditions.

For the sake of brevity, detailed diagrams showing mode transitions associated with the boundary conditions (2.3) and (2.5) is not presented. However, it is worth remarking that the fundamental mode transition associated with (2.3) is remarkably similar to that described in Figure 5. Thus, if we were to consider a transition from a layer with free faces to the layer with fixed faces via a sequence of problems with boundary conditions (2.2) and (2.3), a low-frequency band gap would be formed for both anti-symmetric and symmetric motion. A similar observation would be true for a sequence of problems with boundary conditions (2.4) and (2.5).

The various combinations of boundary conditions considered in this section enable us to formulate a simple physical interpretation of what leads to the creation of an associated low-frequency band gap. A layer with faces that are restrained in the normal direction loses its ability to propagate low-frequency anti-symmetric (bending) waves, see Figure 4. However, the low-frequency symmetric (extensional) motions remain largely unaffected. If the layer's faces are restrained in the tangential direction, the low-frequency extensional motion is not possible, see Figure 5, however low-frequency anti-symmetric motions are still possible. In this case they cannot be characterised as as classical bending, because in this case the long-wave low-frequency limit attains a finite velocity.

\section{Boundaries with a restraint in the normal direction}

The prohibitively large number of asymptotic regimes that are possible in a layer with boundary conditions (2.2)-(2.5) makes it impossible to consider all of the cases in this paper. Thus, we focus our attention on one particular case associated with boundary conditions (2.2). The asymptotic processes necessary to analyse different types of the boundary conditions are all similar to those described in this paper. 


\subsection{Long-wave behaviour of anti-symmetric waves}

In the anti-symmetric case the dispersion relation (3.7) assumes the form

$$
4 q_{1} q_{2} \tanh \left(\eta q_{1}\right)-\left(2+V^{2}\right)^{2} \tanh \left(\eta q_{2}\right)+\frac{q_{2} V^{2} \delta}{\eta}=0
$$

which differs from the classical Rayleigh-Lamb dispersion relation only through the presence of the final term, which specifies the effect of elastically restrained boundaries.

In order to clarify the effect that the boundary elasticity modulus $\delta$ has on the long-wave behaviour of the solution branches, we now proceed to an asymptotic analysis of dispersion relation (5.1). Taking the limit $\eta \rightarrow 0$ yields two families of solutions, namely

$$
\Lambda_{s h}^{a}=\left(n-\frac{1}{2}\right) \pi, \quad n=1,2, \ldots,
$$

and

$$
\kappa \Lambda_{s t}^{a} \tan \left(\frac{\Lambda_{s t}^{a}}{\kappa}\right)=\delta
$$

see also (3.9). The first family (5.2) corresponds to the thickness shear resonance frequencies $\Lambda_{s h}^{a}$ and are independent of $\delta$. The second family, which is implicitly defined by equation (5.3), is associated with thickness stretch resonance frequencies $\Lambda_{s t}^{a}$. The transformations of solution branches of the dispersion relation (5.1) in the long-wave regime are largely dominated by the dependence of $\Lambda_{s t}^{a}$ on $\delta$. In the case of a layer with free faces $(\delta=0)$, equation (5.3) reduces to $\Lambda_{s t}^{a}=\kappa \pi n, n=0,1,2, \ldots$, with $n=0$ corresponding to the low-frequency fundamental mode and $n=1,2, \ldots$ related to thickness stretch resonance frequencies. In the case of a layer with mixed boundary conditions $(\delta=\infty)$, solutions of (5.3) reduce to $\Lambda_{s t}^{a}=\kappa \pi(n-1 / 2), n=1,2, \ldots$ Low-frequency anti-symmetric motions are clearly not possible in this case. It is worth remarking that equation (5.3) may be of practical importance because it allows computing of the boundary elastic modulus $\delta$ directly from an experimentally determined first thickness stretch resonance frequency, also the first thickness resonance for sufficiently small $\delta$. It may also enable quantitative estimates for the stiffness of media that surrounds the layer.

The asymptotic structure of the dispersion relation (5.1) is essentially determined by the two parameters: $\eta$ and $\delta$. In order to establish the principal asymptotic regimes for (5.1), we need to correlate the magnitudes of these parameters. Thus, we seek scalings for $\delta$ of the form

$$
\delta=\delta_{0} \eta^{2 m}, \quad \delta_{0}=O(1)
$$

Case $1(m=1): \delta \eta^{-2} \sim 1$

In this case the dispersion relation (5.1) may be written as

$$
4 q_{1} q_{2} \tanh \left(\eta q_{1}\right)-\left(2+V^{2}\right)^{2} \tanh \left(\eta q_{2}\right)+q_{2} V^{2} \delta_{0} \eta=0 .
$$




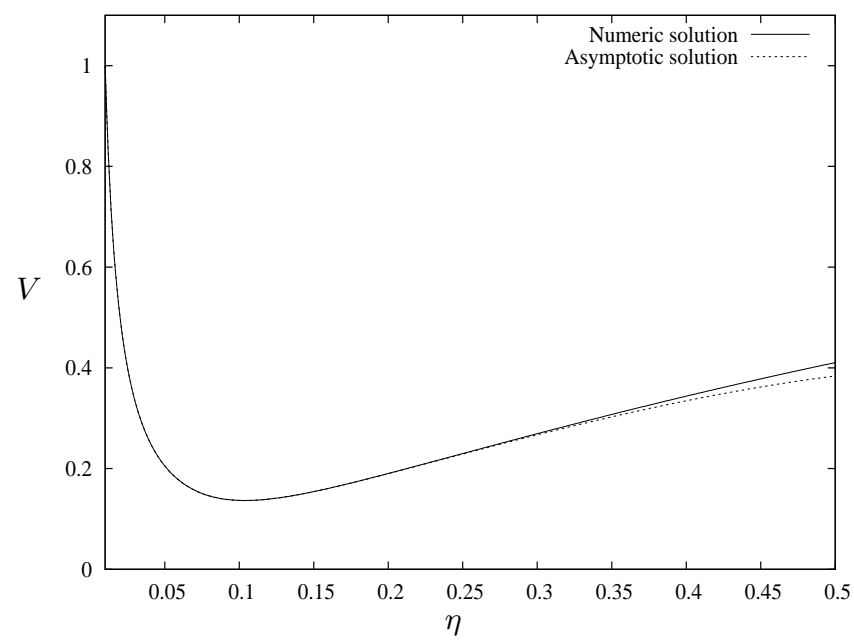

Figure 6: Comparison of the asymptotic solution (5.8) with the numerical solution for $\delta=10^{-4}$ and $\kappa=\sqrt{3}$.

It is possible to identify three asymptotic regimes that can balance (5.5) for long-wave motions. The first possible asymptotic structure is defined by

$$
\tanh \left(\eta q_{1}\right) \sim \eta, \quad \tanh \left(\eta q_{2}\right) \sim \eta, \quad V \sim 1,
$$

and associated with a perturbed low-frequency (fundamental) mode for very small values of $\delta$. Due to the fact that the phase speed is finite within this regime, the hyperbolic tangents can be expanded into the series for small argument. By substituting an asymptotic ansatz of the form

$$
V^{2}=V_{0}^{2}+V_{2}^{2} \eta^{2}+V_{4}^{2} \eta^{4}+O\left(\eta^{6}\right),
$$

into the dispersion relation (5.1) and equating coefficients at every order of $\eta^{2}$ we may obtain the unknown constants $V_{0}, V_{2}, V_{4}$. The resulting third order asymptotic approximation for the phase speed is given by

$$
\begin{aligned}
V^{2} & =\delta_{0}+\left(\frac{4}{3}-\delta_{0}-\frac{\left(\delta_{0}-2\right)^{2}}{3 \kappa^{2}}\right) \eta^{2} \\
& +\left(\frac{37}{15} \delta_{0}-\frac{8}{15} \delta_{0}^{2}-\frac{12}{5}+\frac{\left(57 \delta_{0}-94\right)\left(\delta_{0}-2\right)}{45 \kappa^{2}}+\frac{4\left(\delta_{0}-5\right)\left(\delta_{0}-2\right)^{2}}{45 \kappa^{4}}\right) \eta^{4}+O\left(\eta^{6}\right)
\end{aligned}
$$

Figure 6 demonstrates excellent performance of the asymptotic approximation (5.8) when compared with the exact numerical solution over a wide range of $\eta$. This approximation provides an important insight into the way in which fundamental bending mode degenerates into a harmonic under the influence of elastically constrained boundary.

The second asymptotic regime that is dominated by thickness shear resonance frequencies 
$\Lambda_{s h}^{a}$ is reached when

$$
\tanh \left(\eta q_{1}\right) \sim V^{2}, \quad \tanh \left(\eta q_{2}\right) \sim 1, \quad V \sim \eta^{-1},
$$

this being possible when the argument of hyperbolic tangent tanh $\left(\eta q_{1}\right)$ is near to $\mathrm{i}\left(n-\frac{1}{2}\right) \pi$. Thus, we may expand this argument into power a series for small $\eta$, namely

$$
q_{1} \eta=\mathrm{i} \Lambda_{1}^{a}+\mathrm{i} \phi_{2} \eta^{2}+\mathrm{i} \phi_{4} \eta^{4}+O\left(\eta^{6}\right)
$$

in which we introduced thickness shear resonance frequencies $\Lambda_{1}^{a}=\left(n-\frac{1}{2}\right) \pi, n=1,2, \ldots$, and parameters $\phi_{2}$ and $\phi_{4}$ are (in general) $O(1)$ unknown quantities. The corresponding expansion for $\tanh \left(\eta q_{1}\right)$ is given by

$$
\tanh \left(\eta q_{1}\right)=-\frac{\mathrm{i}}{\eta^{2} \phi_{2}}+\frac{\mathrm{i} \phi_{4}}{\left(\phi_{2}\right)^{2}}+O\left(\eta^{2}\right) .
$$

We may now use (3.4) to obtain corresponding approximations for all required functions in (5.5), thus

$$
\begin{gathered}
V=\frac{\Lambda_{1}^{a}}{\eta}+\frac{2 \Lambda_{1}^{a} \phi_{2}+1}{2 \Lambda_{1}^{a}} \eta+O\left(\eta^{3}\right), \quad q_{2}=\frac{\mathrm{i} \Lambda_{1}^{a}}{\kappa \eta}+O(\eta), \\
\tanh \left(\eta q_{2}\right)=\mathrm{i} \tan \left(\frac{\Lambda_{1}^{a}}{\kappa}\right)+O\left(\eta^{2}\right) .
\end{gathered}
$$

Substituting expansions (5.10)-(5.13) back into the dispersion relation (5.5), we obtain expressions for $\phi_{2}$ and $\phi_{4}$ in the form

$$
\phi_{2}=\frac{4}{\kappa\left(\Lambda_{1}^{a}\right)^{2} \tan \left(\frac{\Lambda_{1}^{a}}{\kappa}\right)}, \quad \phi_{4}=\frac{\left(5-\kappa^{2}\right)}{2\left(\Lambda_{1}^{a}\right)^{2}} \phi_{2}+\frac{\left(\left(\Lambda_{1}^{a}\right)^{2} \delta_{0}-8\right)}{4 \Lambda_{1}^{a}} \phi_{2}^{2} .
$$

It is now possible to obtain the third order approximation for scaled frequency in the form

$$
\Omega^{2}=\left(\Lambda_{1}^{a}\right)^{2}+\left(1+2 \Lambda_{1}^{a} \phi_{2}\right) \eta^{2}+\left(\frac{\left(5-\kappa^{2}\right)}{\Lambda_{1}^{a}} \phi_{2}+\frac{\left(\left(\Lambda_{1}^{a}\right)^{2} \delta_{0}-6\right)}{2} \phi_{2}^{2}\right) \eta^{4}+O\left(\eta^{6}\right) .
$$

We present in Figure 7 a comparison of the asymptotic expansion (5.15) with the numerical solution in the vicinity of the first two shear thickness resonance frequencies. Both numerical and asymptotic solution show excellent agreement over a surprisingly large wave number region. The accuracy improves for higher harmonic numbers, which is associated with decrease in the numerical value of $\phi_{2}$ which has a $\left(\Lambda_{1}^{a}\right)^{-2}$ factor.

The last asymptotic regime capable of satisfying the dispersion relation (5.5) is associated with the thickness stretch resonance frequencies $\Lambda_{s t}^{a}$ and may be identified by

$$
\tanh \left(\eta q_{1}\right) \sim 1, \quad \tanh \left(\eta q_{2}\right) \sim V^{-2}, \quad V \sim \eta^{-1} .
$$

The derivation of an appropriate asymptotic expansion is very similar to the previously considered case for the shear thickness resonances, with the exception that in this case the 


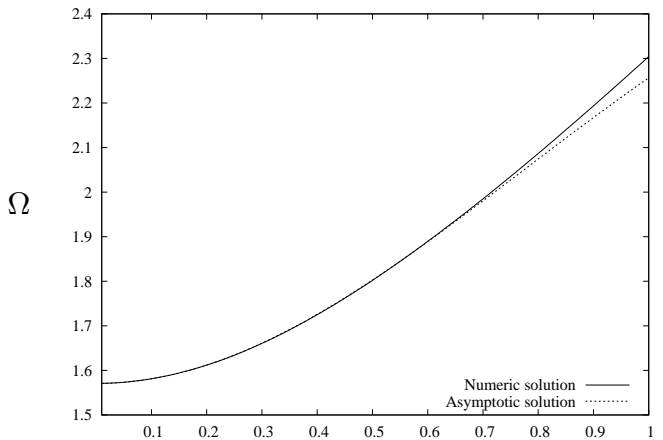

(a)

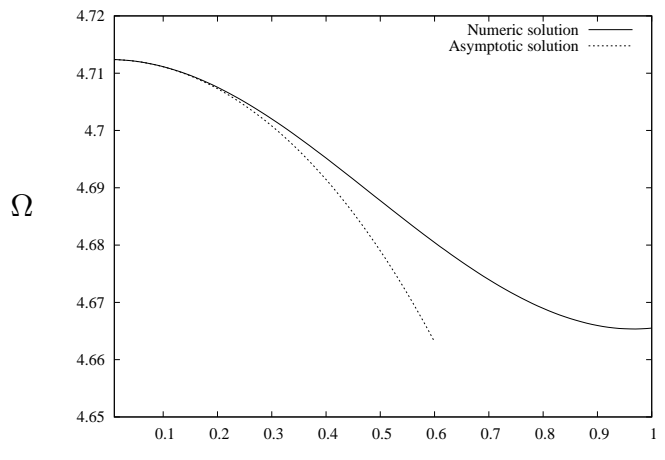

(b)

$\eta$

Figure 7: Comparison of the asymptotic solution (5.15) with the numerical solution for the first (a) and second (b) $S H^{a}$ harmonics for $\delta=10^{-4}$ and $\kappa=\sqrt{3}$.

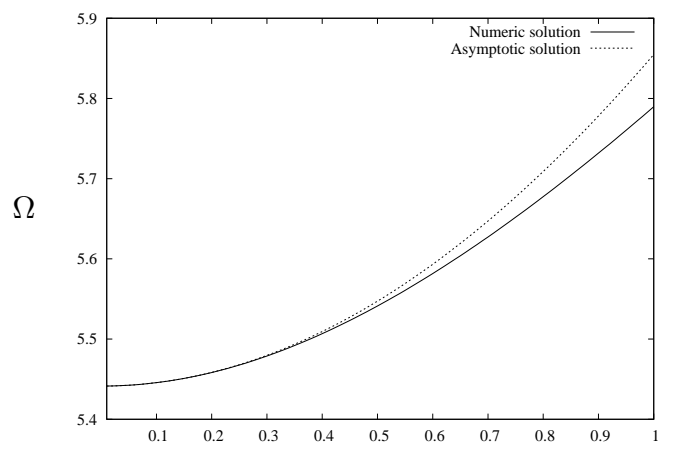

(a)

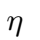

(b)

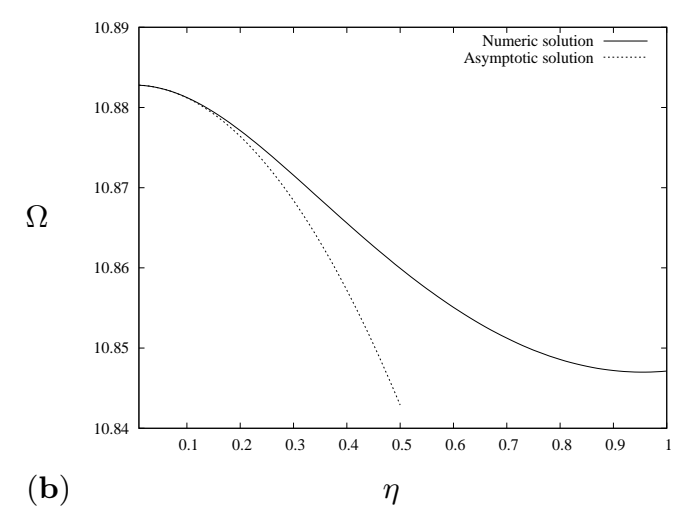

Figure 8: Comparison of the asymptotic solution (5.17) with the numerical solution for the first (a) and second (b) $S H^{a}$ harmonics for $\delta=10^{-4}$ and $\kappa=\sqrt{3}$. 
cut-off frequencies are given by $\Lambda_{2}^{a}=\kappa n \pi, n=1,2, \ldots$ The corresponding approximation for the scaled frequency has the form

$$
\Omega^{2}=\left(\Lambda_{2}^{a}\right)^{2}+\left(\kappa^{2}+\frac{2\left(\Lambda_{2}^{a} \delta_{0}-4 \tan \left(\Lambda_{2}^{a}\right)\right)}{\Lambda_{2}^{a}}\right) \eta^{2}+\frac{\left(\Lambda_{2}^{a} \delta_{0}-4 \tan \left(\Lambda_{2}^{a}\right)\right)^{2}}{\left(\Lambda_{2}^{a}\right)^{4}} \eta^{4}+O\left(\eta^{6}\right) .
$$

Figure 8 demonstrates that good agreement is observed when comparing asymptotic solution (5.17) with the numerical solution.

Case $2(m=-1): \delta \eta^{-2} \sim 1$

Low-frequency motions are not possible for large values of $\delta$, so we only need to consider the long-wave behaviour of two families on harmonics, namely thickness shear and thickness stretch resonance modes. Consequently, the corresponding dispersion relation

$$
4 q_{1} q_{2} \tanh \left(\eta q_{1}\right)-\left(2+V^{2}\right)^{2} \tanh \left(\eta q_{2}\right)+q_{2} V^{2} \frac{\delta_{0}}{\eta^{3}}=0,
$$

supports two asymptotic regimes. The first results in thickness shear resonance modes with the following asymptotic structure

$$
\tanh \left(\eta q_{1}\right) \sim V^{4}, \quad \tanh \left(\eta q_{2}\right) \sim 1, \quad V \sim \eta^{-1}
$$

The derivation of the approximation for scaled frequency is quite similar to Case 1, with the result given by

$$
\Omega^{2}=\left(\Lambda_{1}^{a}\right)^{2}+\eta^{2}-\frac{8 \eta^{4}}{\delta_{0}}+O\left(\eta^{6}\right) .
$$

The simplicity of (5.20) is related to the particularly simple analytical structure of hyperbolic solution branches of the problem with mixed boundary conditions, see [1]. As can be seen on Figure 9(a) the asymptotic and numerical solutions are almost indistinguishable for all harmonics over a large range of wave numbers.

The second asymptotic regime for Case 2 is characterized by the following relative orders of relevant functions

$$
\tanh \left(\eta q_{1}\right) \sim 1, \quad \tanh \left(\eta q_{2}\right) \sim V^{2}, \quad V \sim \eta^{-1}
$$

The associated third-order asymptotic expansion for scaled frequency is given in this case by

$$
\Omega^{2}=\left(\Lambda_{4}^{a}\right)^{2}+\left(\kappa^{2}-\frac{2 \kappa^{2}\left(\Lambda_{4}^{a}\right)^{2}}{\delta_{0}}\right) \eta^{2}+\left(\frac{3 \kappa^{4}\left(\Lambda_{4}^{a}\right)^{2}}{\delta_{0}^{2}}-\frac{2 \kappa^{2}\left(\kappa^{2}-4\right)}{\delta_{0}}\right) \eta^{4}+O\left(\eta^{6}\right) .
$$

where $\Lambda_{4}^{a}=(n-1 / 2) \kappa \pi$. Excellent agreement with the numerical solution is observed within Figure 9(b) for first two thickness stretch resonance harmonics when $\delta=10000$. 


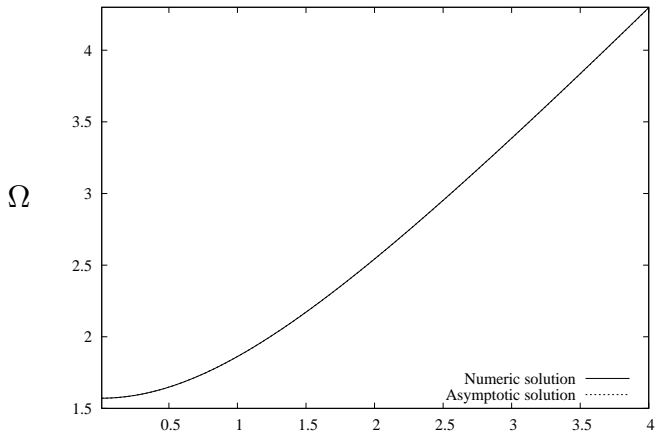

(a)

$\eta$

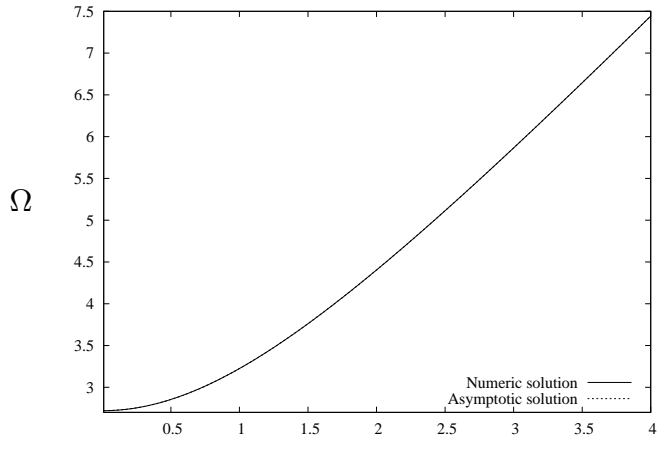

(b)

$\eta$

Figure 9: Comparison asymptotic solution (5.20) (a) and (5.22) (b) for the first harmonic with numerical solutions for $\delta=10000$ and $\kappa=\sqrt{3}$.

Case $3(m=0): \delta \sim 1$

When $\delta \sim O(1)$ long-wave low-frequency motions is not possible; only high-frequency motion is therefore possible within the long wave regime. Similarly to the high-frequency subcases, these modes are dominated by thickness shear and thickness stretch resonances, i.e. motion must be within the vicinities of $\Lambda_{s h}^{a}$ and $\Lambda_{s t}^{a}$, respectively. The frequencies $\Lambda_{s h}^{a}$ remain unaffected by changes in $\delta$, nevertheless, frequencies $\Lambda_{s t}^{a}$ increase as $\delta$ increases. At a certain value of $\delta=\delta_{*}$ a member of $\Lambda_{s t}^{a}$ may coincide with a member of $\Lambda_{s t}^{a}$. In this case the asymptotic structure of both associated modes change and special quasi-linear expansions need to be developed, see $[9,14]$. While both standard and quasi-linear asymptotics are simple to derive and well-understood now, their applicability ranges are defined by certain asymptotic conditions that may be difficult to implement in a purely numerical context. Additionally, the accuracy of standard long-wave high-frequency asymptotics typically deteriorates long before the quasi-linear expansion can be used. Thus, it will be useful to develop a procedure capable of producing uniform asymptotic expansions for long-wave high-frequency motion applicable to this case. The appropriate expansions will now be presented for both types of thickness resonance frequencies.

\section{Thickness shear expansion}

Within the long-wave regime the dispersion relation (5.1) may be represented as

$$
O\left(V^{2}\right) \tanh \left(\eta q_{1}\right)+O\left(V^{4}\right) \tanh \left(\eta q_{2}\right)+O\left(V^{4}\right)=0
$$

which is balanced for thickness shear resonance frequencies by making tanh $\left(\eta q_{1}\right)$ sufficiently large. To be more precise, it is usually necessary to assume that

$$
\tanh \left(\eta q_{1}\right) \sim V^{2}, \quad \tanh \left(\eta q_{2}\right) \sim 1
$$


so that the argument of $\tanh \left(\eta q_{1}\right)$ is dominated by i $\Lambda_{3}^{a}$, where $\Lambda_{3}^{a}=(n-1 / 2) \pi, n=1,2, \ldots$. However, in the close vicinity of certain $\delta=\delta_{*}$ such that

$$
\delta_{*}=\kappa \Lambda_{3}^{a} \tan \frac{\Lambda_{3}^{a}}{\kappa},
$$

the asymptotic regime described by (5.24) switches to

$$
\tanh \left(\eta q_{1}\right) \sim V, \quad \tanh \left(\eta q_{2}\right)-\frac{\delta_{*}}{\kappa \Lambda_{3}^{a}} \sim V^{-1} .
$$

In order to represent both of these cases within a single asymptotic anzats we seek the $\operatorname{argument}$ of $\tanh \left(\eta q_{1}\right)$ in the following modified form

$$
\eta q_{1}=\mathrm{i}\left(\Lambda_{3}^{a}+\eta \epsilon\right), \quad \tanh \left(\eta q_{1}\right)=-\frac{\mathrm{i}}{\epsilon \eta}+O(1),
$$

where the function $\epsilon=\epsilon(\eta)$ and we assume that it may vary between $O(\eta)$ and $O(1)$. By carefully keeping all of the terms required in both asymptotic regimes, it is possible to establish that

$$
\begin{aligned}
\Omega & =\Lambda_{3}^{a}+\epsilon \eta+\frac{\eta^{2}}{2 \Lambda_{3}^{a}}+O\left(\epsilon \eta^{3}\right), \\
q_{2} & =\frac{\mathrm{i} \Lambda_{3}^{a}}{\kappa \eta}+\frac{\mathrm{i} \epsilon}{\kappa}-\frac{\mathrm{i}\left(\kappa^{2}-1\right)^{2} \eta}{2 \kappa \Lambda_{3}^{a}}+O\left(\epsilon \eta^{2}\right), \\
\tanh \left(\eta q_{2}\right) & =\mathrm{i} \tan \left(\frac{\Lambda_{3}^{a}}{\kappa}\right)+\frac{\mathrm{i} \epsilon \eta}{\kappa \cos ^{2}\left(\frac{\Lambda_{3}^{a}}{\kappa}\right)}+O\left(\eta^{2}\right) .
\end{aligned}
$$

If these expansions are inserted back into the dispersion relation (5.1), we obtain a quadratic equation for $\epsilon$, given by

$$
\left[3 \delta-4 \kappa \Lambda_{3}^{a} \tan \left(\frac{\Lambda_{3}^{a}}{\kappa}\right)-\frac{\Lambda_{3}^{a 2}}{\cos ^{2}\left(\frac{\Lambda_{3}^{a}}{\kappa}\right)}\right] \epsilon^{2}+\Lambda_{3}^{a}\left[\delta-\kappa \Lambda_{3}^{a} \tan \left(\frac{\Lambda_{3}^{a}}{\kappa}\right)\right] \frac{\epsilon}{\eta}+4=0 .
$$

In view of the fact that we sought our expansion in the vicinity of a single cut-off frequency $\Lambda_{3}^{a}$, only one root of (5.29) describes a thickness shear mode. The second root only becomes relevant in the vicinity of $\delta=\delta_{*}$, where it corresponds to the coupled thickness stretch mode. If we denote the smaller root of (5.29) $\epsilon=\epsilon_{1}$ and the larger root $\epsilon=\epsilon_{2}$, the following simple condition enables selecting the correct root:

$$
\epsilon= \begin{cases}\epsilon_{2}, & \delta<\delta_{*} \\ \epsilon_{1}, & \delta>\delta_{*} .\end{cases}
$$

It is worth reiterating that when $\delta=\delta_{*}$ the notions of thickness shear and thickness stretch become meaningless and both solutions of (5.29) become asymptotically consistent. The corresponding expansion for the scaled frequency may be written as

$$
\Omega=\Lambda_{3}^{a}+\epsilon \eta+\frac{\eta^{2}}{2 \Lambda_{3}^{a}}+O\left(\epsilon \eta^{3}\right) .
$$



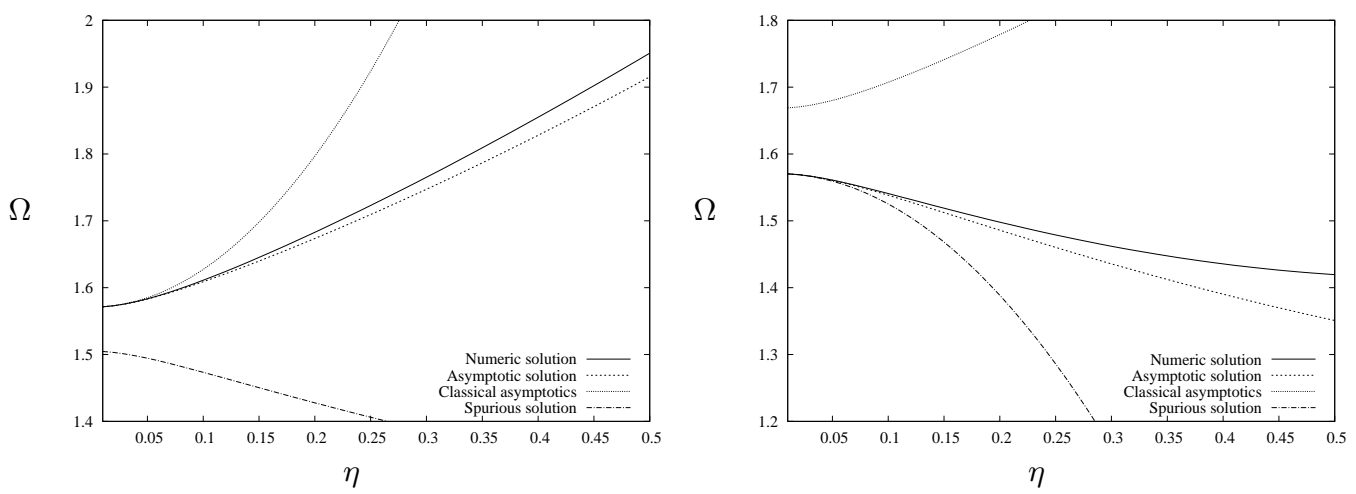

Figure 10: Comparison of uniform asymptotic solution (5.31) and numerical solution for the first SH-mode when $\delta=3$ and $\kappa=\sqrt{3}$. Also presented standard non-uniform asymptotic and the spurious branch corresponding to incorrect choice of $\epsilon$.

Comparison of the uniform asymptotic expansion (5.31) and exact numerical solutions for $\delta=3$ and $\kappa=\sqrt{3}$ is shown in Figure 10. The standard non-uniform asymptotic is also presented to demonstrate its inaccuracy even when $\delta$ is relatively far from $\delta_{*} \approx 3.4775$.

\section{Thickness stretch expansion}

The procedure for deriving an asymptotic expansion in the vicinity of thickness stretch resonance frequencies is similar, except that in this case the cut-off frequencies $\Lambda_{4}^{a}$ are $\delta$ dependent and found from the implicit relationship

$$
\kappa \Lambda_{4}^{a} \tan \left(\frac{\Lambda_{4}^{a}}{\kappa}\right)=\delta .
$$

The standard (non-uniform) asymptotic expansion would rely upon the scaling

$$
\tanh \left(\eta q_{1}\right) \sim 1, \quad \tanh \left(\eta q_{2}\right)-\frac{\delta}{\kappa \Lambda_{4}^{a}} \sim V^{-2} .
$$

However, when $\delta \sim \delta_{*}$, see (5.25), this structure changes to

$$
\tanh \left(\eta q_{1}\right) \sim V, \quad \tanh \left(\eta q_{2}\right)-\frac{\delta_{*}}{\kappa \Lambda_{4}^{a}} \sim V^{-1},
$$

where (5.34) is clearly equivalent to (5.26).

Similarly, as with the thickness shear case, we seek a uniform asymptotic expansion that includes both cases (5.33) and (5.34). This is done by introducing the following anzats

$$
\eta q_{2}=\mathrm{i}\left(\frac{\Lambda_{4}^{a}}{\kappa}+\eta \epsilon\right), \quad \tanh \left(\eta q_{2}\right)=\frac{\mathrm{i} \delta}{\kappa \Lambda_{4}^{a}}+\mathrm{i}\left(\frac{\delta^{2}}{\kappa^{2}\left(\Lambda_{4}^{a}\right)^{2}}+1\right) \epsilon \eta,
$$

in which the condition (5.3) has been used and we again assume that $\epsilon=\epsilon(\eta)$, which may vary between $O(\eta)$ and $O(1)$. Thus, the general form of our asymptotic expansion is given 

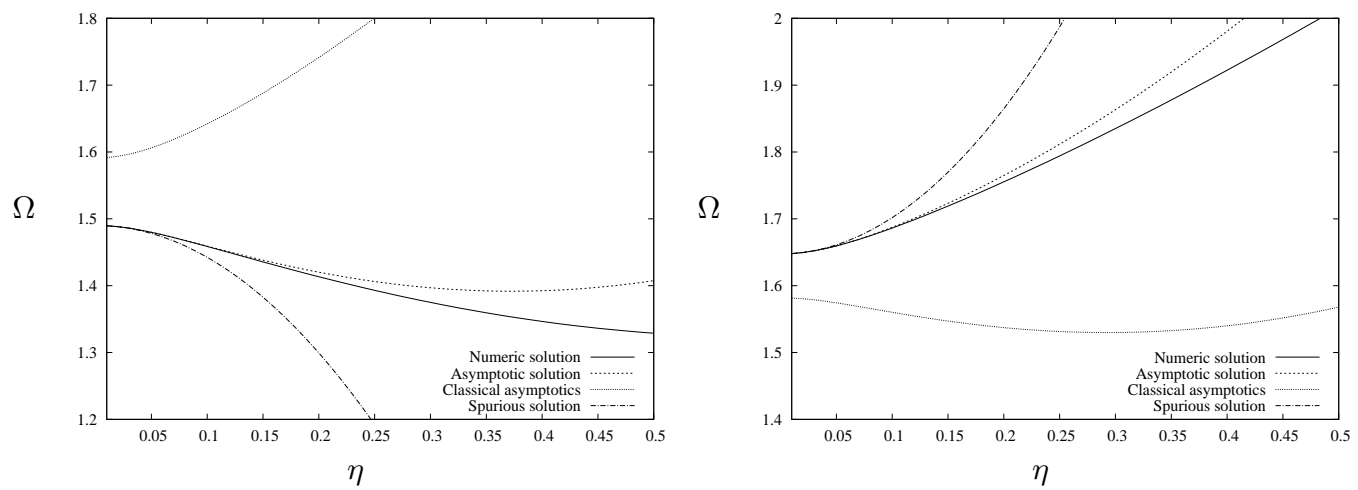

Figure 11: Comparison of uniform asymptotic solution (5.39) and numerical solution for the former fundamental when $\delta=4$ and $\kappa=\sqrt{3}$. Also presented standard non-uniform asymptotic and the spurious branch corresponding to incorrect choice of $\epsilon$.

by

$$
\Omega=\Lambda_{4}^{a}+\kappa \epsilon \eta+\frac{\kappa^{2} \eta^{2}}{2 \Lambda_{4}^{a}} .
$$

By keeping in mind our assumptions on $\epsilon$, it is possible to obtain the following expansions

$$
q_{1}=\frac{i \Lambda_{4}^{a}}{\eta}+i \kappa \epsilon, \quad \tanh \left(\eta q_{1}\right)=\frac{i \tan \left(\Lambda_{4}^{a}\right)}{1-\kappa \tan \left(\Lambda_{4}^{a}\right) \epsilon \eta} .
$$

If these are inserted into dispersion relation (5.1), we obtain the following quadratic equation for $\epsilon$

$$
\left[A \tan \left(\Lambda_{4}^{a}\right)-\frac{4 A-\kappa^{2} \delta}{\Lambda_{4}^{a}}\right] \epsilon^{2}-\frac{A \epsilon}{\kappa \eta}-4 \tan \left(\Lambda_{4}^{a}\right)-\frac{\delta\left(\kappa^{2}-4\right)}{\Lambda_{4}^{a}}=0 .
$$

in which $A=\delta^{2}+\kappa^{2} \delta+\kappa^{2}\left(\Lambda_{4}^{a}\right)^{2}$. If we denote the smaller root of (5.37) $\epsilon=\epsilon_{1}$ and the larger root as $\epsilon=\epsilon_{2}$, then the rule to choose the correct root is given by

$$
\epsilon= \begin{cases}\epsilon_{1}, & \delta<\delta_{*} ; \\ \epsilon_{2}, & \delta>\delta_{*} .\end{cases}
$$

Once again, when $\delta=\delta_{*}$ both roots result in a correct asymptotic expansion. The corresponding approximation for scaled frequency has the form

$$
\Omega=\Lambda_{4}^{a}+\kappa \epsilon \eta+\frac{\kappa^{2} \eta^{2}}{2 \Lambda_{4}^{a}} .
$$

It is easy to verify that when $\delta=\delta_{*}$ both asymptotic expansions (5.31) and (5.39) result in the same expression. Comparison of the uniform asymptotic solution (5.39) with the numerical solutions for $\delta=4$ is shown on the Figure 11. The performance of (5.31) (or (5.39)) in the case when $\delta=\delta_{*}$ is illustrated in Figure 12. Both solution branches demonstrate good agreement with the exact numerical solution. 


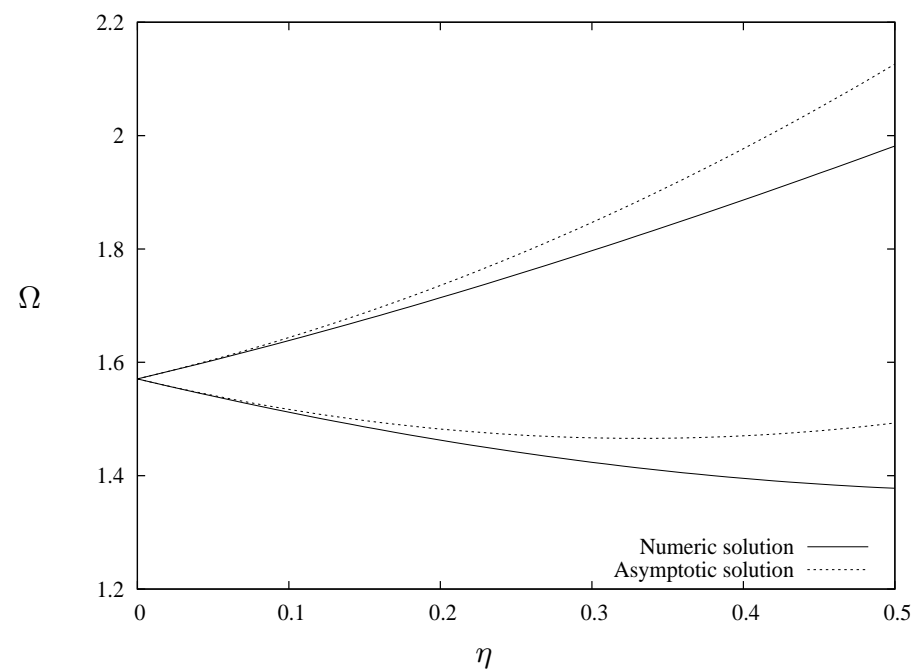

Figure 12: Comparison of the uniform asymptotic solution (5.39) with the numerical solution for the special case with $\delta=\delta_{*} \approx 3.4775$ and $\kappa=\sqrt{3}$.

\subsection{Long wave analysis of symmetric waves}

Analysis of symmetric modes in the case of normally restrained boundaries is similar to the analysis presented in respect of anti-symmetric motion. Thus, in this Section the results of the corresponding asymptotic analysis are presented without derivations. The relevant dispersion relation has the form

$$
4 q_{1} q_{2} \operatorname{coth}\left(\eta q_{1}\right)-\left(2+V^{2}\right)^{2} \operatorname{coth}\left(\eta q_{2}\right)+\frac{q_{2} V^{2} \delta}{\eta}=0 .
$$

The long-wave limit of (5.40) has two types of solutions, $c f$. (3.10). The first is given by

$$
\Lambda_{s h}^{s}=n \pi, \quad n=0,1,2, \ldots,
$$

and the second is implicitly defined by the transcendental equation

$$
-\kappa \Lambda_{s t}^{s} \cot \left(\frac{\Lambda_{s t}^{s}}{\kappa}\right)=\delta .
$$

The symmetric fundamental mode originates from (5.41) when $n=0$. Its long-wave limit does not depend on $\delta$, which is also true for the remainder of the frequencies (5.41), associated with thickness shear resonances. We remark that the thickness stretch frequencies $\Lambda_{s t}^{s}$ do depend on $\delta$.

As commented in Section 4, the symmetric fundamental mode does not change its asymptotic structure due to the effect of normal restraint on the layer faces. Thus, the following long-wave low-frequency expansion for the phase speed of fundamental mode

$$
V^{2}=\frac{(4+\delta) \kappa^{2}-4}{\delta+\kappa^{2}}+\frac{\left(\kappa^{2}-2\right)^{2}\left(4-4 \kappa^{2}-\delta \kappa^{2}\right)}{3\left(\delta+\kappa^{2}\right)^{3}} \eta^{2},
$$


is valid for all values of $\delta$. Nonetheless, the analysis of high-frequency solutions of (5.40) again requires re-scaling of the parameter $\delta$ as described by (5.4), with the associated expansions presented in the following cases.

Case $1(m=1): \delta \eta^{-2} \sim 1$

The asymptotic expansion for symmetric modes related to thickness shear has the form

$$
\Omega^{2}=\left(\Lambda_{1}^{s}\right)^{2}+\left(1-\frac{8}{\kappa \Lambda_{1}^{s} \cot \left(\frac{\Lambda_{1}^{s}}{\kappa}\right)}\right) \eta^{2}+\left(\frac{4\left(\kappa^{2}-5\right)}{\kappa\left(\Lambda_{1}^{s}\right)^{3} \cot \left(\frac{\Lambda_{1}^{s}}{\kappa}\right)}+\frac{8\left(\left(\Lambda_{1}^{s}\right)^{2} \delta_{0}-6\right)}{\kappa^{2}\left(\Lambda_{1}^{a}\right)^{4} \cot ^{2}\left(\frac{\Lambda_{1}^{s}}{\kappa}\right)}\right) \eta^{4},
$$

where $\Lambda_{1}^{s}=n \pi, n=1,2, \ldots$ The modes associated with thickness stretch have the following asymptotic expansion

$$
\Omega^{2}=\left(\Lambda_{2}^{s}\right)^{2}+\left(\kappa^{2}+\frac{2\left(\Lambda_{2}^{s} \delta_{0}+4 \cot \left(\Lambda_{2}^{s}\right)\right)}{\Lambda_{2}^{s}}\right) \eta^{2}+\frac{\left(\Lambda_{2}^{s} \delta_{0}+4 \cot \left(\Lambda_{2}^{s}\right)\right)^{2}}{\left(\Lambda_{2}^{s}\right)^{4}} \eta^{4},
$$

in which $\Lambda_{2}^{s}=(n-1 / 2) \kappa \pi, n=1,2, \ldots$

Case $2(m=-1): \delta \eta^{-2} \sim 1$

The asymptotics for large $\delta$ were found to have the same expansions as in the anti-symmetric case, namely (5.20) and (5.22) for modes related to thickness shear and thickness stretch, respectively, in which $\Lambda_{1}^{a}$ must be replaced by $\Lambda_{1}^{s}$, and $\Lambda_{4}^{a}$ by $\Lambda_{4}^{s}=\kappa n \pi$.

Case $3(m=0): \delta \sim 1$

In this case we obtained uniform asymptotics near both thickness shear and thickness stretch resonance frequencies by using the same techniques as were developed in analysis of the antisymmetric modes. The expansions for scaled frequency are identical to (5.31) and (5.39), which should be used with slightly modified equations for $\epsilon$. For the thickness shear modes $\epsilon$ is found from

$$
\left[3 \delta+4 \kappa \Lambda_{3}^{s} \cot \left(\frac{\Lambda_{3}^{s}}{\kappa}\right)-\frac{\Lambda_{3}^{s 2}}{\sin \left(\frac{\Lambda_{3}^{s}}{\kappa}\right)^{2}}\right] \epsilon^{2}+\Lambda_{3}^{s}\left[\delta+\kappa \Lambda_{3}^{s} \cot \left(\frac{\Lambda_{3}^{s}}{\kappa}\right)\right] \frac{\epsilon}{\eta}+4=0,
$$

whereas for thickness shear from

$$
\left[A \cot \left(\Lambda_{4}^{s}\right)+\frac{4 A-\kappa^{2} \delta}{\Lambda_{4}^{s}}\right] \epsilon^{2}+\frac{A \epsilon}{\kappa \eta}-4 \cot \left(\Lambda_{4}^{s}\right)+\frac{\delta\left(\kappa^{2}-4\right)}{\Lambda_{4}^{s}}=0 .
$$

in which $\Lambda_{3}^{s}=n \pi, \Lambda_{4}^{s}$ satisfies relation (5.42), and $A$ is defined immediately after (5.37). 


\section{Short-wave limits and the surface wave speed}

It is evident from the dispersion relations (3.7) and (3.8) that the influence of elastically restrained boundaries diminishes for shorter waves, as $\eta \rightarrow \infty$. In the subsonic regime, when $V<1$, both $q_{1}$ and $q_{2}$ are real, see (3.3). This means that also

$$
\lim _{\eta \rightarrow \infty} \tanh \left(\eta q_{1}\right)=\lim _{\eta \rightarrow \infty} \tanh \left(\eta q_{2}\right)=1
$$

Therefore, for sufficiently short waves both anti-symmetric and symmetric dispersion relations both tend to

$$
4 q_{1} q_{2}-\left(q_{1}^{2}+1\right)^{2}+\left(q_{1} \frac{\delta_{1}}{\alpha_{1}}+q_{2} \frac{\delta_{2}}{\alpha_{2}}\right) \frac{V^{2}}{\eta}+\frac{\delta_{1} \delta_{2}}{\alpha_{1} \alpha_{2}} \frac{\left(1-q_{1} q_{2}\right)}{\eta^{2}}=0 .
$$

It is possible to show that (6.2) is, in fact, the secular equation for surface waves propagating in an elastic isotropic half-space with the boundary conditions (2.6)-(2.7).

Let us first assume that $\alpha_{1} \neq 0$ and $\alpha_{2} \neq 0$. The first two terms of (6.2) describe the leading-order behaviour at large $\eta$ and can be recognised as the classical Rayleigh secular equation. Thus, the short-wave limit of the fundamental branches for the layers with elastically restrained boundaries is always the Rayleigh wave speed $V_{R}$, that is, the solution of

$$
4 q_{1} q_{2}-\left(q_{1}^{2}+1\right)^{2} \equiv 4 \sqrt{1-V_{R}^{2}} \sqrt{1-V_{R}^{2} / \kappa^{2}}-\left(2-V_{R}^{2}\right)^{2}=0 .
$$

For large but finite $\eta$ relation (6.2) describes physical dispersion of surface waves travelling along layer faces that is caused by interaction with the elastic restraints. This dispersion may be described by the two-term expansion

$$
\begin{aligned}
V^{2}=V_{R}^{2}+\frac{16\left(1-V_{R}^{2}\right)\left(\kappa^{2}-V_{R}^{2}\right)}{\left(2-V_{R}^{2}\right)\left(\left(1-V_{R}^{2}\right)\left(8+\kappa^{2} V_{R}^{2}\right)+\left(7 \kappa^{2}-8\right) V_{R}^{2}\right)} \\
\quad \times \frac{1}{\eta}\left(\frac{\delta_{1}}{\alpha_{1}} \sqrt{1-V_{R}^{2}}+\frac{\delta_{2}}{\alpha_{2}} \sqrt{1-V_{R}^{2} / \kappa^{2}}\right)+O\left(\eta^{-2}\right) .
\end{aligned}
$$

The sign of the $O\left(\eta^{-1}\right)$ term is positive for $\kappa^{2}>8 / 7$, i.e., in particular, for all positive Poisson ratios. It is possible to verify numerically that it remains positive for all $\nu \in(-1,1 / 2)$. Therefore, the velocity of surface wave grows as the wave number decreases. At some point $\eta_{*}$, the velocity reaches 1 and the surface wave degenerates into a shear wave. However, the asymptotic approximation (6.2) is no longer valid when $V=1$ (because $q_{1}$ vanishes). Thus, in order to determine $\eta_{*}$ it is necessary to return back to the original dispersion relations and analyse the limiting case for $V=1$. The appropriate expansion of (3.7) leads to an implicit relationship

$$
\left(1-\frac{\delta_{1} \delta_{2}}{\alpha_{1} \alpha_{2}} \frac{1}{\eta_{*}^{2}}\right) \tanh \left(\eta_{*} \frac{\sqrt{\kappa^{2}-1}}{\kappa}\right)=\frac{\delta_{2}}{\alpha_{2}} \frac{\sqrt{\kappa^{2}-1}}{\eta_{*} \kappa} .
$$




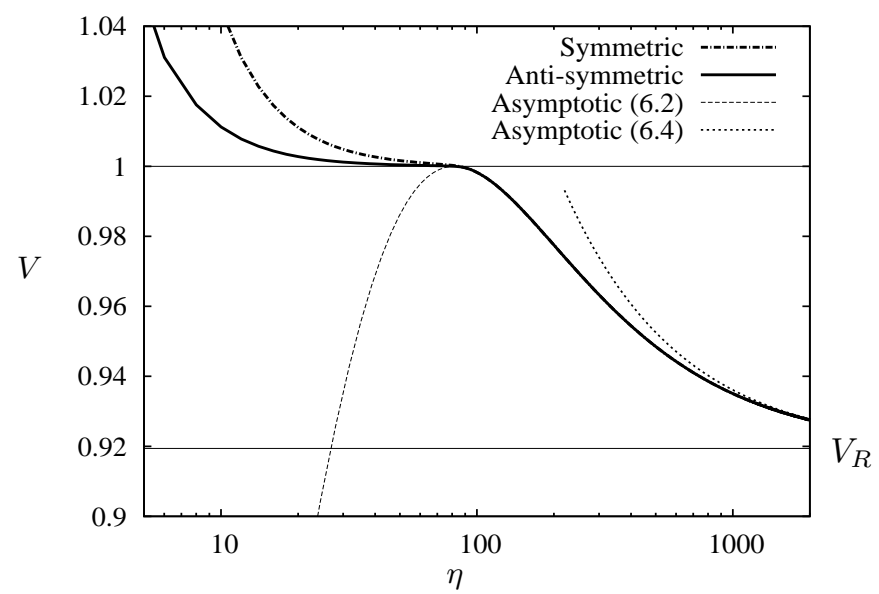

Figure 13: Anti-symmetric and symmetric fundamental modes shown together with surface and shear wave speeds, as well as asymptotic expansion (6.4) for $\kappa=\sqrt{3}$ and $\delta=100$.

In the case of symmetric dispersion relation (3.8) the corresponding limit is

$$
\left(1-\frac{\delta_{1}}{\alpha_{1}} \frac{1}{\eta_{*}^{2}}-\frac{\delta_{1} \delta_{2}}{\alpha_{1} \alpha_{2}} \frac{1}{\eta_{*}^{2}}\right) \operatorname{coth}\left(\eta_{*} \frac{\sqrt{\kappa^{2}-1}}{\kappa}\right)=\left(4+\frac{\delta_{2}}{\alpha_{2}}-\frac{\delta_{1} \delta_{2}}{\alpha_{1} \alpha_{2}} \frac{1}{\eta_{*}^{2}}\right) \frac{\sqrt{\kappa^{2}-1}}{\eta_{*} \kappa} .
$$

Expressions (6.5) and (6.6) become particularly simple when $\alpha_{1}=\alpha_{2}=1, \delta_{1}=0$ and $\delta_{2}=\delta$. In this case, as $\delta$ grows larger, the values of $\eta_{*}$ for both anti-symmetric and symmetric cases become indistinguishable so that $\eta_{*} \sim \delta \sqrt{\kappa^{2}-1} / \kappa$.

For example, Figure 13 demonstrates the short-wave behaviour of both anti-symmetric and symmetric fundamental modes for $\delta=100$. Expansion (6.4) works well for large wave numbers, but as $\eta$ approaches $\eta_{*}$ the error becomes significant. After crossing the line $V=1$ both fundamental modes monotonically tend to their respective long-wave limits. It is interesting to note that while (6.2) provides very accurate approximation of both fundamental modes when $\eta>\eta_{*}$, their behaviour becomes substantially different for $\eta<\eta_{*}$, even when $\eta_{*}$ is large. The behaviour at $\eta<\eta_{*}$ is clearly not associated with two coupled surface waves travelling on the opposite sides of the layer.

Finally, in the case when $\alpha_{1}=0$ and/or $\alpha_{2}=0$, the appropriate short-wave limit of (6.2) does no longer correspond to the Rayleigh wave. Furthermore, it can be shown that this limit possesses no subsonic solutions, which is simply a reflection of the fact that surface wave cannot propagate along a fixed boundary.

\section{Acknowledgement}

The work of the first author was supported by an INTAS grant, YSF/06-10000014-5790. This award is very gratefully acknowledged. 


\section{References}

[1] K. F. Graff. Wave Motion in Elastic Solids. Dover Publications, Inc., New York, 1991.

[2] J. D. Kaplunov. Long-wave vibrations of a thin-walled body with fixed faces. Quarterly Journal of Mechanics and Applied Mathematics, 48(3):311-327, 1995.

[3] J. D. Kaplunov, L. Y. Kossovich, and E. V. Nolde. Dynamics of Thin Walled Elastic Bodies. Academic Press, New York, 1998.

[4] J. D. Kaplunov, L. Yu. Kossovich, and G. A. Rogerson. Direct asymptotic integration of the equations of transversely isotropic elasticity for a plate near cut-off frequencies. The Quarterly Journal of Mechanics and Applied Mathematics, 53(2):323-341, 2000.

[5] J. D. Kaplunov, E. V. Nolde, and G. A. Rogerson. A low frequency model for dynamic motion in pre-stressed incompressible elastic structures. Proceedings of the Royal Society of London, Series A, 456(2003):2589-2610, 2000.

[6] L. Y. Kossovitch, R. R. Moukhomodiarov, and G. A. Rogerson. Analysis of the dispersion relation for an incompressible transversely isotropic elastic plate. Acta Mechanica, 153(1-2):89-111, 2002.

[7] H. Lamb. On waves in an elastic plate. Proceedings of the Royal Society of London, Series A, 93(648):114-128, 1917.

[8] R. D. Mindlin. Waves and vibrations in isotropic, elastic plates. In J. N. Goodier and N. J. Hoff, editors, Structural Mechanics, pages 199-232. Pergamon Press, Inc., Oxford, New York, 1960.

[9] E. V. Nolde, L. A. Prikazchikova, and G. A. Rogerson. Dispersion of small amplitude waves in a pre-stressed, compressible elastic plate. Journal of Elasticity, 75(1):1-29, 2004.

[10] A. V. Pichugin and G. A. Rogerson. A two-dimensional model for extensional motion of a pre-stressed incompressible elastic layer near cut-off frequencies. IMA Journal of Applied Mathematics, 66(4):357-385, 2001.

[11] O. Poncelet, A. L. Shuvalov, and J. Kaplunov. Approximation of the flexural velocity branch in plates. International Journal of Solids and Structures, 43(21):6329-6346, 2006.

[12] J. W. S. Rayleigh. On the free vibrations of an infinite plate of homogeneous isotropic elastic matter. Proceedings of the London Mathematical Society, 20:225-234, 1889. 
[13] A. L. Shuvalov. On the theory of wave propagation in anisotropic plates. Proceedings of the Royal Society, Series A, 456(2001):2197-2222, 2000.

[14] A. L. Shuvalov and O. Poncelet. On the backward lamb waves near thickness resonances in anisotropic plates. International Journal of Solids and Structures, 45(11-12):3430$3448,2008$.

[15] M. F. Werby and H. Überall. The analysis and interpretation of some special properties of higher order symmetric lamb waves: The case for plates. Journal of the Acoustical Society of America, 111(6):2686-2691, 2002. 